\title{
POISSON REGRESSION OF DAMAGE PRODUCT SALES USING MCMC
}

\author{
Reny Rian Marliana ${ }^{1 \ddagger}$, Septiadi Padmadisastra² \\ ${ }^{1}$ Department of Informatics, Sumedang School of IT, Indonesia, renyrianmarliana@gmail.com \\ 2Department of Statistics, Padjadjaran University, Indonesia, s_padmadisastra@yahoo.com \\ Fcorresponding author \\ Indonesian Journal of Statistics and Its Applications (elSSN:2599-0802) \\ Vol 2 No 1 (2018), 1 - 12
}

Copyright ( 2018 Reny Rian Marliana and Septiadi Padmadisastra. This is an open-access article distributed under the Creative Commons Attribution License, which permits unrestricted use, distribution, and reproduction in any medium, provided the original work is properly cited.

\begin{abstract}
In this paper a model for the number of "damage" product sales is studied. The product sales are run into underreporting counts, caused by a delay on input process of the system called sales cycle. The goal of the study is to estimate the parameters of the regression model of product sales on an explanatory variable. It is the actual number of product sales. The model used is a mixture of the Poisson and the Binomial distributions. The parameters of the regression model are estimated by a Bayesian approach and Markov Chain Monte Carlo simulation using Gibbs sampling algorithm. The results of estimation clearly showed a gap between undamage product sales and the actual number. The gap is the number of damaged product sales.
\end{abstract}

Keywords: bayesian, gibbs sampling, mcmc, underreported

\section{Introduction}

Misreporting counts can occur in any system of reporting. Li et al. (2003), in his regression model, misreporting occurs when an individual report on the number of observed events is different from the actual values (as cited in Pararai, 2010). Thus, misreporting counts divided into two, underreporting and over reporting counts. Underreporting is a problem in data collection, when the counting of observed events, for some reason incomplete (Neubauer et al., 2011). Underreported counts occur when the number of observed events, reported smaller than the actual number of

"Received Des 2017; Accepted Apr 2018; Published online on Apr 2018 
occurrences. Instead, over reported counts occur when the number of observed events, reported more than of the actual number of occurrences.

In this research, underreported counts occurred as a result of delay on input process of the number of product sales repeatedly. In this system (see Figure 1), the input process of the number of product sales will directly reduce the stock number of the products in the counter. That would indirectly lead to over reporting the stock number the products in the counter. This is a major factor that caused errors on the next production's plan or in the distribution of products. In order to reduce the risks, the actual number of product sales is need to be estimated.

The consequence of the underreporting counts is the number of product sales reported is only part of the actual number of product sales. There are a number of product sales which are unreported. It means that a product sold at a counter will have two possible treatments from the administrator i.e. inputted into the system or not. An opportunity for a product sold at a counter inputted to the system called probability of reported. This probability has a value ranging from $0-1$. It is also known that the actual number of product sales in a counter within a month was random and ranged between 0-21 pieces with an average of 4 pieces per month. Both of the information can be considered as a prior information that can be used in the estimation of the actual number of product sales.

The actual number of product sales is influenced by the activity of selling the product itself. As cited in Sinaga (2013), one of the factors that affects sales activities is market conditions (Basu, 2005). The market conditions can be seen as a rate of product sales at its counter. Thus, to estimate the actual number of product sales, need an analysis that can describe the relationship between the number of products sales (underreported) with rate of product sales involving both the prior information before.

So far modeling of the count data using Poisson regression model and binomial regression models. However, both models can be used if the count data is considered accurately reported. As cited in Papadatos (2005), models for under-reported counts were first introduced in Moran's characterization (1952) and Rao-Rubin condition (1964). Then, several researchers have developed a model for underreported counts, including the Poisson regression model for underreported counts developed by Winkelmann (1996), Mukhopadhyay (1997) developed the negative binomial regression model for underreported counts (as cited in Pararai, 2010) and the generalized Poisson-Poisson mixture models which can be used for misreporting counts (under, over and accurately reported) developed by Pararai (2010).

In estimating the model parameters, Pararai (2010) used classical statistical approach which only use sample data information through the maximum likelihood method. Meanwhile, Winkelmann (1996) used a Bayesian statistical approach in estimating the model parameters. 


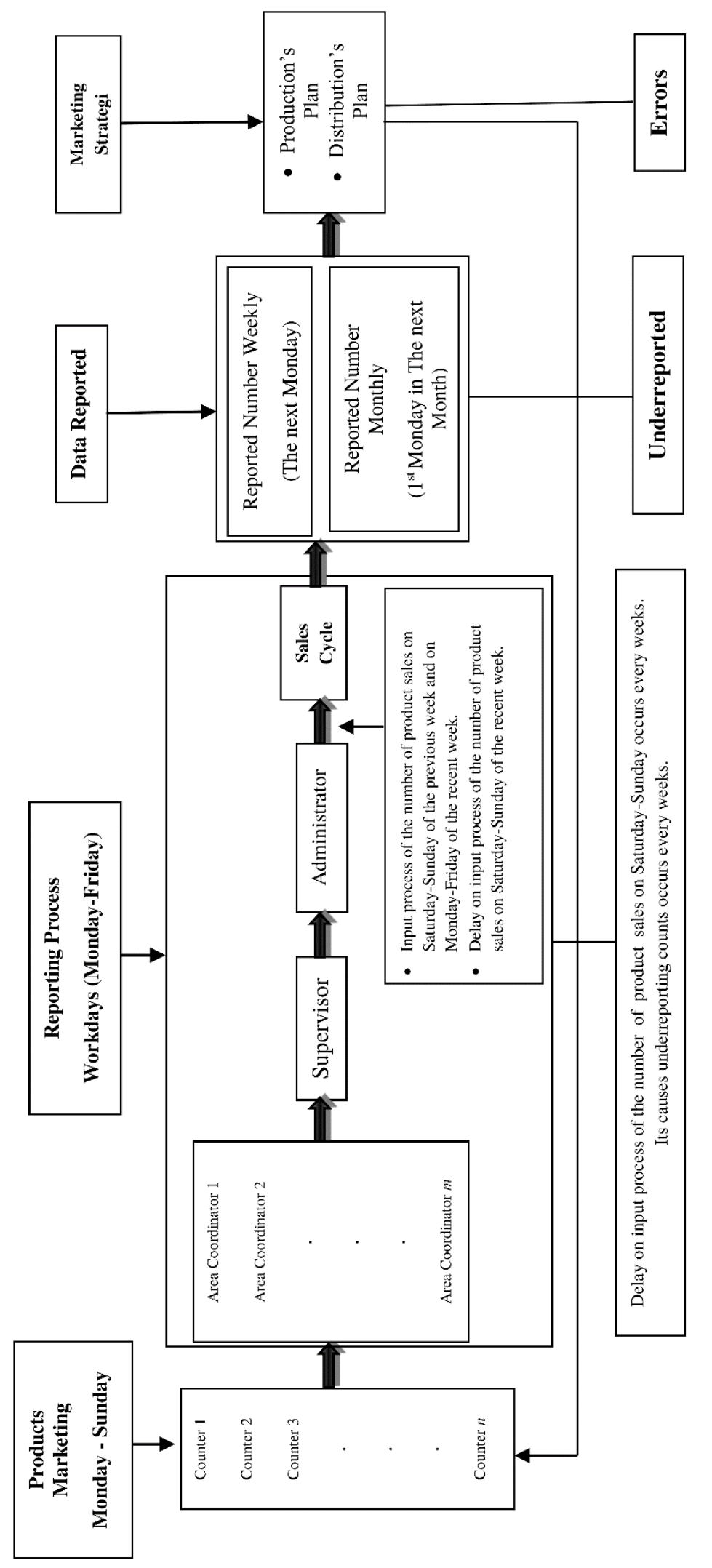

Figure 1: Reporting System of A Garment Company 


\section{Poisson Model for Underreported Counts}

Moran (1952) introduced a characterization of underreported counts (as cited in Papadatos, 2005). It is stated that if $N_{1}$ and $N_{2}$ are non-degenerate independent random variables and its values are non-negative and if the conditional distribution of $N_{1}\left(N_{1}+N_{2}=n\right)$ is binomial and its parameter is $n \in N=\{0,1, \cdots\}$ with success probability $p \in[0,1]$ for some $n \in N, P\left[N_{1}+N_{2}=n\right]>0$ then the distributions of $N_{1}, N_{2}$ and $\left(N_{1}+N_{2}=n\right)$ are Poisson. For some $i \in N, P\left[N_{1}=i\right]>0$ and $P\left[N_{2}=i\right]>0$.

Another form of Moran's (1952) characterization was introduced by Rao and Rubin (1964), independence of $N_{1}$ and $N_{2}$ called as Rao-Rubin condition. For some $p \in[0,1]$ , then Rao-Rubin condition implies that $N_{1}$ and $N_{2}$ follow a Poisson distribution with parameter $\lambda p$ and $\lambda(1-p)$ for some $\lambda>0$.

\section{Poisson Regression Model for Underreported Counts}

Poisson regression model for underreported counts which is developed by Winkelmann (1996) was also a mixture of binomial distribution and Poisson distribution.

Let $Y_{i}^{*}$ as an actual number of observed events in a specific time for individual $i$. Assume that $Y_{i}^{*}$ depends on $\mathbf{x}_{i}$ and follows a Poisson distribution with parameter $\lambda_{i}=\exp \left[\mathbf{x}_{i}^{\prime} \boldsymbol{\beta}\right]$. Let $Y_{i}$ as the reported number of observed events in a specific time for individual $i$ and follows a binomial distribution with parameters $Y_{i}^{*}$ and $p$ (probability of reported).

Underreported counts occur if $Y_{i}^{*}>Y_{i}$ so the marginal distribution of $Y_{i}$ is a Poisson regression model with parameter $\lambda_{i} p=\exp \left[\mathbf{x}_{i}^{\prime} \boldsymbol{\beta}\right] \cdot p$.

Parameter $p_{i}$ cannot be treated as a fixed parameter since the model is singular with $n+k$ parameter and $n$ data points (Winkelmann, 1996). To solve this problem, Winkelmann consider the parameter $p_{i}$ as a random variabel which follows a certain distribution. Further, Winkelmann (1996) obtained the posterior distribution of $y^{*}, p$ and $\boldsymbol{\beta}$ as:

$$
\underbrace{P\left(y^{*}, p, \boldsymbol{\beta} \mid y, \mathbf{x}\right)}_{\text {posterior }} \propto \underbrace{P\left(y \mid y^{*}, p\right)}_{\text {likelihood }} \times \underbrace{P\left(y^{*} \mid \boldsymbol{\beta}\right) \times f(\boldsymbol{\beta}) \times f(p)}_{\text {prior }}
$$

Drapper \& Guttman (1971) assume that the parameters $N$ and $p$ are independent random variables, where $N$ follows a discrete uniform distribution and $p$ follows a beta distribution (as cited in Moreno, 1998). Meanwhile, Raftery (1988) assumes that $N$ follows a Poisson distribution with parameter $\lambda$ and $p$ follows a uniform distribution (as cited in Moreno, 1998). Winkelmann (1996) assumes that $N$ or $Y^{*}$ follows a Poisson distribution with parameter $\lambda=\exp \left[\mathbf{x}^{\prime} \boldsymbol{\beta}\right]$ where the $\boldsymbol{\beta}$ assumed follows a normally distribution with parameter $\mu$ and $\sigma$, and $p$ assumed follows a uniform distribution. The Posterior distribution of $Y_{i}^{*}$ is (Winkelmann,1996) : 


$$
\begin{aligned}
P\left(Y_{i}^{*}, \boldsymbol{\beta}, p_{i} \mid Y_{i}\right) \propto & P\left(Y_{i} \mid Y_{i}^{*}, p_{i}\right) P\left(Y_{i}^{*} \mid \boldsymbol{\beta}\right) f(\boldsymbol{\beta}) f\left(p_{i}\right) \\
& \propto \exp \left[-\frac{1}{2}(\boldsymbol{\beta}-\boldsymbol{\mu})^{\prime} \Sigma^{-1}(\boldsymbol{\beta}-\boldsymbol{\mu})\right] \\
& \cdot \prod_{i=1}^{n} \exp \left[\left(y_{i}^{*} \cdot \mathbf{x}_{i}^{\prime} \boldsymbol{\beta}\right)-\exp \left[\mathbf{x}_{i}^{\prime} \boldsymbol{\beta}\right]\right] \frac{p_{i}^{y_{i}}\left(1-p_{i}\right)^{y_{i}^{*}-y_{i}}}{\left(y_{i}^{*}-y_{i}\right) ! y_{i} !}
\end{aligned}
$$

with $Y_{i}^{*}=0,1,2, \cdots ; Y_{i}=0,1,2, \cdots ; 0 \leq p_{i} \leq 1 ;-\infty \leq \boldsymbol{\beta} \leq \infty ; i=1,2, \cdots, n$

\section{Markov Chain Monte Carlo (MCMC)}

MCMC is done by drafting markov chain that converges quickly on the posterior distribution. MCMC generate sample data of parameter $\theta$ which has a spesific distribution through an algorithm and done iteratively (the value of each step depends on the previous step). Gibbs sampling is one of the MCMC algortihm which usually used. Gibss sampling can be applied if the conditional distribution of each parameter is known.

Estimation of the paramater of the model using MCMC will depend on the determination of the burn in period, which is done in line with determination the convergence of the algorithm using trace plot, autocorrelation plot and ergodic mean plot.

Base from the posterior distribution developed by Winkelmann, it is obvious that it is difficult to determine the kind of posterior distribution. To facilitate the parameter estimation, Markov Chain Monte Carlo (MCMC) simulation with full conditional distribution is used as given in the Winkelmann (1996). The MCMC Algorithm is as follows :

1. Set the initial value of each parameters $Y^{*(0)}, p^{(0)}$ and $\boldsymbol{\beta}^{(0)}$

2. Set the number of iteration $T$

3. For $t=1,2, \ldots, T$ repeat the following steps :

a. Generate new candidate of $Y_{i}^{*(t)}$ from $Y_{i}^{*} \mid \boldsymbol{\beta}^{(t-1)}, p_{i}^{(t-1)}, Y_{i}$

b. Generate new candidate of $p_{i}^{(t)}$ from $p_{i} \mid Y_{i}, Y_{i}^{*(t)}$

c. Generate new candidate of $\boldsymbol{\beta}^{(t)}$ from $\boldsymbol{\beta} \mid Y_{i}^{*(t)}, Y_{i}$ using Random-Walks algorithm with $\boldsymbol{\beta}^{(t)}=\boldsymbol{\beta}^{(t-1)}+\left[\tau \mathbf{V}_{\beta}^{-1 / 2} \mathbf{z}\right]$ and $\mathbf{z} \sim N_{D}(\mathbf{0}, \mathbf{I})$

4. Update the values of each parameter using the values obtained by the simulation.

5. Check the convergence of the algorithm using Autocorrelation plot, Trace plot and Ergodic mean plot

6. Determine the burn in period.

7. From the simulated values after the burn-in until the last iteration count:: 
a. The average value and standard deviation of the parameter $\boldsymbol{\beta}$

b. The average value and standard deviation of the parameter $p_{i}$ for $i=1,2, \cdots, n$

c. The average value and standard deviation of the parameter $Y_{i}^{*}$ for $i=1,2, \cdots, n$

\section{Results and Discussion}

The data used in this study is product sales at 108 counters in August 2013 of a garment company in Bandung City, Indonesia. These counters belong to the group of regions that have the highest sales target compared with other counters across of Indonesia. The rate of product sales of these counters was also the highest compared with others. This causes the risk of delay on input process is very high, while the products distributions must run every week and production plans run every month (see Figure 1). This is a major factor that lead in increasing the errors on the marketing strategies (se Figure 1). In order to reduce the risks, the actual number of product sales is need to be estimated.

Count variable, reported number of product sales has values between 1-26 pieces with an average of 5.44 and standard deviation 4.57 . While the independent variable is the rate of products sales in each counter and divided into 4 categories such as very high, high, low and very low. Therefore, the regression model of the actual number of product sales involved 3 dummy variables. MCMC simulation performed with 5000 iterations and the algorithm before. To check the convergence of the series we use trace plot, autocorrelation plot and ergodic mean plot.

All the trace plot (simulation $p$, simulation $Y^{*}$ and simulation $\beta$ ) did not show a certain pattern. The autocorrelation plots (simulation $p$, simulation $Y^{*}$ and simulation $\boldsymbol{\beta}$ ) showed there are not an autocorrelation between two iterations. Ergodic mean plots showed that ergodic mean of the sample values of parameter $p, Y^{*}$ and $\boldsymbol{\beta}$ are stabilize after the first 3000 iterations. Then, the convergence of the simulation for parameter $p$ and $Y^{*}$ and regression parameter $\boldsymbol{\beta}$ has been achieved with the burn in period of 3000 first iterations.

From the simulations, the results of the regression parameter estimation can be seen in Table 1 below:

Table 1: Regression Parameters Estimate

\begin{tabular}{ccc}
\hline Parameter & Estimator & Stdev. \\
\hline$\beta_{0}$ & 1.9929 & 0.4709 \\
$\beta_{1}$ & 1.0721 & 0.4792 \\
$\beta_{2}$ & 0.3876 & 0.4779 \\
$\beta_{3}$ & 0.1774 & 0.4843 \\
\hline
\end{tabular}

It can be stated that the average of the actual number of product sales in August 2013 is a function of : 


$$
\hat{\lambda}_{i}=E\left[Y_{i}^{*} \mid X_{i}\right]=e^{\left[1,9929+1,0721 D_{1 i}+0,3876 D_{2 i}+0,1774 D_{3 i}\right]} .
$$

Therefore, based on the category rate of products sales in each counters, the estimates of the average of the actual number of the product sales in August 2013 can be seen in Table 2 .

The mean values of product sales, obtained from the above regression equation, presented in the above table. It can be seen that the average of the actual number of the product sales in August 2013 at the counter with the rate of product sales very high is 21 pieces, at the counter with the rate of product sales high is 11 pieces, at the counter with the rate of product sales low is 9 pieces and at the counter with the rate of product sales very low is 7 pieces.

Tabel 2: The Estimators of The Mean of The Actual Number of The Product Sales

\begin{tabular}{cc}
\hline The Rate Of Product & $\hat{\lambda}_{i}=E\left[Y_{i}^{*} \mid X_{i}\right]$ \\
Sales & 21 \\
Very High & 11 \\
High & 9 \\
Low & 7 \\
Very Low &
\end{tabular}

To determine the possibility occurences of underreporting counts, we compared the estimated value of the average of the actual number of the product sales (see Table 2) with the number of product sales recorded in the report (underreported counts). The results (see Table 3 ) show that the product sales of $87.04 \%$ counters from 108 are run into underreporting counts. It means that the risk of error in the production's plans, product distributions or in other marketing strategies will be high.

Tabel 3: The Comparison of The Estimators of The Average of The Actual Number of The Product Sales on Product Sales Report (Underreported Counts)

\begin{tabular}{cccccc}
\hline $\begin{array}{c}\text { The Rate Of Product } \\
\text { Sales }\end{array}$ & $\hat{\lambda}_{i}>y_{i}$ & $\hat{\lambda}_{i}<y_{i}$ & $\hat{\lambda}_{i}=y_{i}$ & Total & $\begin{array}{c}\text { Percentage of } \\
\text { Underreporting }\end{array}$ \\
\hline Very High & 12 & 2 & - & 14 & $85.71 \%$ \\
High & 48 & 5 & 3 & 56 & $85.71 \%$ \\
Low & 32 & 2 & 2 & 36 & $88.89 \%$ \\
Very Low & 2 & - & - & 2 & $100 \%$ \\
Total & $\mathbf{9 4}$ & $\mathbf{9}$ & $\mathbf{5}$ & $\mathbf{1 0 8}$ & $\mathbf{8 7 . 0 4 \%}$ \\
\hline
\end{tabular}

Meanwhile, the results of the estimation of the actual number of the product sales or $Y^{*}$ and the probability for a product sold reported (inputted to the system) or $p$ based on the category the rate of products sales in each counter are in the following Table 4, Table 5, Table 6 and Table 7.

From Table 4, we get that the average of the estimators of the actual number of the product sales at a counter with the rate of product sales very high is ranging between 17-26 pieces with a gap of 8-18 pieces compared to the number of the product sales listed in the report (obtained from the system). The mean percentage of underreporting counts of product sales is $60.03 \%$ or ranged from $10.71 \%$ to $90.75 \%$. 
Tabel 4 : The Estimators of Model Parameter at Counter with The Very High Rate of Product Sales

\begin{tabular}{ccccccccc}
\hline Counter & $y_{\boldsymbol{i}}$ & $\widehat{y_{l}^{*}}$ & $\operatorname{std}\left(\widehat{y_{l}}\right)$ & $\widehat{p_{l}^{*}}$ & $\operatorname{std}\left(\widehat{p_{l}^{*}}\right)$ & $\widehat{q_{l}^{*}} x 100 \%$ & $\begin{array}{c}\widehat{y_{l}^{*}} \pm \\
s t d\left(\widehat{y_{l}}\right)\end{array}$ & $\begin{array}{c}{\left[\widehat{y_{l}^{*}} \pm\right.} \\
\left.s t d\left(\hat{y}_{l}\right)\right]-y_{i}\end{array}$ \\
\hline Counter 1 & 15 & 21 & 4 & 0.7122 & 0.1533 & $28.78 \%$ & $17-26$ & $2-11$ \\
Counter 2 & 1 & 21 & 5 & 0.0957 & 0.0696 & $90.43 \%$ & $15-26$ & $14-25$ \\
Counter 3 & 23 & 26 & 3 & 0.8648 & 0.1052 & $13.52 \%$ & $23-29$ & $0-6$ \\
Counter 4 & 11 & 21 & 5 & 0.5583 & 0.1638 & $44.17 \%$ & $16-26$ & $5-15$ \\
Counter 5 & 4 & 21 & 5 & 0.2299 & 0.1037 & $77.01 \%$ & $16-26$ & $12-22$ \\
Counter 6 & 1 & 21 & 5 & 0.0925 & 0.0655 & $90.75 \%$ & $15-26$ & $14-25$ \\
Counter 7 & 8 & 21 & 5 & 0.4234 & 0.1435 & $57.66 \%$ & $15-26$ & $7-18$ \\
Counter 8 & 5 & 21 & 5 & 0.2804 & 0.1196 & $71.96 \%$ & $16-26$ & $11-21$ \\
Counter 9 & 13 & 21 & 5 & 0.6445 & 0.1633 & $35.55 \%$ & $16-26$ & $3-13$ \\
Counter 10 & 4 & 20 & 5 & 0.2362 & 0.1087 & $76.38 \%$ & $15-26$ & $11-22$ \\
Counter 11 & 26 & 28 & 3 & 0.8929 & 0.0857 & $10.71 \%$ & $26-31$ & $0-5$ \\
Counter 12 & 1 & 20 & 5 & 0.0959 & 0.0681 & $90.41 \%$ & $15-26$ & $14-25$ \\
Counter 13 & 3 & 21 & 5 & 0.1852 & 0.096 & $81.48 \%$ & $15-26$ & $12-23$ \\
Counter 14 & 5 & 21 & 5 & 0.2839 & 0.1181 & $71.61 \%$ & $15-26$ & $10-21$ \\
Average & $\mathbf{9}$ & $\mathbf{2 2}$ & $\mathbf{5}$ & $\mathbf{0 . 3 9 9 7}$ & $\mathbf{0 . 1 1 1 7}$ & $\mathbf{6 0 . 0 3 \%}$ & $\mathbf{1 7 - 2 6}$ & $\mathbf{8 - 1 8}$ \\
\hline
\end{tabular}

From Table 5, we get that the average of the estimators of the actual number of the product sales at a counter with the rate of product sales high is ranging between 8-14 pieces with a gap of 3-8 pieces compared to the number of the product sales listed in the report (obtained from the system). The mean percentage of underreporting counts of product sales is $50.29 \%$ or ranged from $9.09 \%$ to $81.87 \%$.

Tabel 5 : The Estimators of Model Parameter at Counter with The High Rate of Product Sales

\begin{tabular}{ccccccccc}
\hline Counter & $y_{i}$ & $\widehat{y_{l}^{*}}$ & $s t d\left(\widehat{y_{l}}\right)$ & $\widehat{p_{l}^{*}}$ & $s t d\left(\widehat{p_{l}^{*}}\right)$ & $\widehat{q_{l}^{*}} x 100 \%$ & $\begin{array}{c}\widehat{y_{l}^{*}} \pm \\
s t d\left(\widehat{\hat{y}_{l}}\right)\end{array}$ & $\begin{array}{c}{\left[\widehat{y_{i}^{*}} \pm\right.} \\
\left.\operatorname{std}\left(\widehat{y}_{l}\right)\right]-y_{i}\end{array}$ \\
\hline Counter 1 & 11 & 13 & 2 & 0.8147 & 0.1324 & $18.53 \%$ & $11-15$ & $0-4$ \\
Counter 2 & 5 & 10 & 3 & 0.526 & 0.1937 & $47.40 \%$ & $7-14$ & $2-9$ \\
Counter 3 & 18 & 19 & 1 & 0.9091 & 0.0797 & $9.09 \%$ & $18-20$ & $0-2$ \\
Counter 4 & 1 & 10 & 3 & 0.1813 & 0.1273 & $81.87 \%$ & $7-13$ & $6-12$ \\
Counter 5 & 6 & 10 & 3 & 0.5947 & 0.1885 & $40.53 \%$ & $7-13$ & $1-7$ \\
Counter 6 & 12 & 14 & 2 & 0.8328 & 0.1262 & $16.72 \%$ & $12-16$ & $0-4$ \\
Counter 7 & 2 & 10 & 3 & 0.2778 & 0.1599 & $72.22 \%$ & $6-13$ & $4-11$ \\
Counter 8 & 5 & 10 & 3 & 0.5255 & 0.193 & $47.45 \%$ & $7-13$ & $2-8$ \\
Counter 9 & 5 & 10 & 3 & 0.5294 & 0.1921 & $47.06 \%$ & $7-13$ & $2-8$ \\
Counter 10 & 1 & 10 & 3 & 0.1852 & 0.1315 & $81.48 \%$ & $6-13$ & $5-12$ \\
Counter 11 & 3 & 10 & 3 & 0.366 & 0.1801 & $63.40 \%$ & $7-13$ & $4-10$ \\
Counter 12 & 3 & 10 & 3 & 0.3612 & 0.177 & $63.88 \%$ & $7-13$ & $4-10$ \\
Counter 13 & 2 & 10 & 3 & 0.2773 & 0.162 & $72.27 \%$ & $6-13$ & $4-11$ \\
Counter 14 & 7 & 11 & 3 & 0.6781 & 0.1884 & $32.19 \%$ & $8-13$ & $1-6$ \\
Counter 15 & 6 & 10 & 3 & 0.6034 & 0.1929 & $39.66 \%$ & $7-13$ & $1-7$ \\
Counter 16 & 11 & 13 & 2 & 0.8143 & 0.1341 & $18.57 \%$ & $11-15$ & $0-4$ \\
\hline
\end{tabular}




\begin{tabular}{|c|c|c|c|c|c|c|c|c|}
\hline Counter & $y_{i}$ & $\widehat{y_{l}^{*}}$ & $\operatorname{std}\left(\widehat{y}_{l}\right)$ & $\widehat{p_{l}^{*}}$ & $\operatorname{std}\left(\widehat{p_{l}^{*}}\right)$ & $\widehat{q_{l}^{*}} \times 100 \%$ & $\begin{array}{c}\widehat{y_{l}^{*}} \pm \\
\operatorname{std}\left(\widehat{y}_{l}\right)\end{array}$ & $\begin{array}{c}{\left[\widehat{y_{l}^{*}} \pm\right.} \\
\left.\operatorname{std}\left(\widehat{y}_{l}\right)\right]-y_{i}\end{array}$ \\
\hline Counter 17 & 13 & 15 & 2 & 0.8484 & 0.1157 & $15.16 \%$ & 13-16 & $0-3$ \\
\hline Counter 18 & 3 & 10 & 3 & 0.3776 & 0.1865 & $62.24 \%$ & $6-13$ & $3-10$ \\
\hline Counter 19 & 3 & 10 & 3 & 0.3544 & 0.1683 & $64.56 \%$ & $7-13$ & $4-10$ \\
\hline Counter 20 & 7 & 11 & 3 & 0.6443 & 0.1831 & $35.57 \%$ & $8-14$ & $1-7$ \\
\hline Counter 21 & 10 & 12 & 2 & 0.7844 & 0.1449 & $21.56 \%$ & $10-15$ & $0-5$ \\
\hline Counter 22 & 14 & 16 & 2 & 0.8652 & 0.1069 & $13.48 \%$ & $14-17$ & $0-3$ \\
\hline Counter 23 & 7 & 11 & 3 & 0.6487 & 0.1872 & $35.13 \%$ & $8-14$ & $1-7$ \\
\hline Counter 24 & 3 & 10 & 3 & 0.3707 & 0.1777 & $62.93 \%$ & $6-13$ & $3-10$ \\
\hline Counter 25 & 6 & 10 & 3 & 0.599 & 0.1941 & $40.10 \%$ & $7-13$ & $1-7$ \\
\hline Counter 26 & 5 & 10 & 3 & 0.5168 & 0.1856 & $48.32 \%$ & $7-14$ & $2-9$ \\
\hline Counter 27 & 11 & 13 & 2 & 0.8085 & 0.1387 & $19.15 \%$ & $11-15$ & $0-4$ \\
\hline Counter 28 & 3 & 10 & 3 & 0.3723 & 0.1823 & $62.77 \%$ & $6-13$ & $3-10$ \\
\hline Counter 29 & 1 & 10 & 3 & 0.1833 & 0.1307 & $81.67 \%$ & $7-13$ & $6-12$ \\
\hline Counter 30 & 2 & 10 & 3 & 0.2747 & 0.1628 & $72.53 \%$ & $7-13$ & $5-11$ \\
\hline Counter 31 & 6 & 11 & 3 & 0.5893 & 0.186 & $41.07 \%$ & $8-14$ & $2-8$ \\
\hline Counter 32 & 10 & 13 & 2 & 0.7666 & 0.1514 & $23.34 \%$ & $10-15$ & $0-5$ \\
\hline Counter 33 & 12 & 14 & 2 & 0.8394 & 0.1216 & $16.06 \%$ & $12-16$ & $0-4$ \\
\hline Counter 34 & 2 & 10 & 3 & 0.2812 & 0.1589 & $71.88 \%$ & $6-13$ & $4-11$ \\
\hline Counter 35 & 8 & 11 & 3 & 0.6985 & 0.1715 & $30.15 \%$ & $9-14$ & $1-6$ \\
\hline Counter 36 & 1 & 10 & 3 & 0.1822 & 0.1252 & $81.78 \%$ & $7-13$ & $6-12$ \\
\hline Counter 37 & 6 & 10 & 3 & 0.5994 & 0.189 & $40.06 \%$ & $7-13$ & $1-7$ \\
\hline Counter 38 & 4 & 10 & 3 & 0.4421 & 0.1903 & $55.79 \%$ & $7-13$ & $3-9$ \\
\hline Counter 39 & 1 & 10 & 3 & 0.185 & 0.1326 & $81.50 \%$ & $7-13$ & $6-12$ \\
\hline Counter 40 & 2 & 10 & 3 & 0.2744 & 0.1582 & $72.56 \%$ & $6-13$ & $4-11$ \\
\hline Counter 41 & 2 & 10 & 3 & 0.273 & 0.1582 & $72.70 \%$ & $6-13$ & $4-11$ \\
\hline Counter 42 & 7 & 11 & 3 & 0.661 & 0.1858 & $33.90 \%$ & $8-13$ & $1-6$ \\
\hline Counter 43 & 2 & 10 & 3 & 0.2775 & 0.1608 & $72.25 \%$ & $7-13$ & $5-11$ \\
\hline Counter 44 & 7 & 11 & 3 & 0.6493 & 0.1855 & $35.07 \%$ & $8-14$ & $1-7$ \\
\hline Counter 45 & 2 & 10 & 3 & 0.2743 & 0.1612 & $72.57 \%$ & $7-13$ & $5-11$ \\
\hline Counter 46 & 2 & 10 & 3 & 0.2821 & 0.1589 & $71.79 \%$ & $6-13$ & $4-11$ \\
\hline Counter 47 & 8 & 11 & 3 & 0.7136 & 0.1718 & $28.64 \%$ & $9-14$ & $1-6$ \\
\hline Counter 48 & 1 & 10 & 3 & 0.1833 & 0.1291 & $81.67 \%$ & $7-13$ & $6-12$ \\
\hline Counter 49 & 4 & 10 & 3 & 0.444 & 0.1847 & $55.60 \%$ & $7-13$ & $3-9$ \\
\hline Counter 50 & 4 & 10 & 3 & 0.4573 & 0.1949 & $54.27 \%$ & $7-13$ & $3-9$ \\
\hline Counter 51 & 2 & 10 & 3 & 0.2671 & 0.1521 & $73.29 \%$ & $7-13$ & $5-11$ \\
\hline Counter 52 & 3 & 10 & 3 & 0.3687 & 0.1827 & $63.13 \%$ & $7-13$ & $4-10$ \\
\hline Counter 53 & 7 & 11 & 3 & 0.6523 & 0.1822 & $34.77 \%$ & $8-14$ & $1-7$ \\
\hline Counter 54 & 3 & 10 & 3 & 0.3651 & 0.1774 & $63.49 \%$ & $7-13$ & $4-10$ \\
\hline Counter 55 & 5 & 10 & 3 & 0.5348 & 0.19 & $46.52 \%$ & $7-13$ & $2-8$ \\
\hline Counter 56 & 4 & 10 & 3 & 0.4511 & 0.1943 & $54.89 \%$ & $7-13$ & $3-9$ \\
\hline Average & 5 & 11 & 3 & 0.4971 & 0.1639 & $50.29 \%$ & 8-14 & $3-8$ \\
\hline
\end{tabular}

From Table 6, we get that the average of the estimators of the actual number of the product sales at a counter with the rate of product sales low is ranging between 6-11 pieces with a gap of 2-7 pieces compared to the number of the product sales listed in 
the report (obtained from the system). The mean percentage of underreporting counts of product sales is $49.20 \%$ or ranged from $11.70 \%$ to $77.46 \%$.

Tabel 6: The Estimators of Model Parameter at Counter with The Low Rate of Product Sales

\begin{tabular}{|c|c|c|c|c|c|c|c|c|}
\hline Counter & $y_{i}$ & $\widehat{y_{l}^{*}}$ & $\operatorname{std}\left(\widehat{y}_{l}\right)$ & $\widehat{p_{l}^{*}}$ & $\operatorname{std}\left(\widehat{p_{l}^{*}}\right)$ & $\widehat{q_{l}^{*}} \times 100 \%$ & $\begin{array}{c}\widehat{y_{l}^{*} \pm} \\
\operatorname{std}\left(\widehat{y}_{l}\right)\end{array}$ & $\begin{array}{c}{\left[\widehat{y_{l}^{*}} \pm\right.} \\
\left.\operatorname{std}\left(\widehat{y_{l}}\right)\right]-y_{i}\end{array}$ \\
\hline Counter 1 & 1 & 8 & 3 & 0.2313 & 0.1586 & $76.87 \%$ & $5-11$ & 4-10 \\
\hline Counter 2 & 5 & 8 & 3 & 0.6114 & 0.202 & $38.86 \%$ & $6-11$ & $1-6$ \\
\hline Counter 3 & 9 & 11 & 2 & 0.7924 & 0.1471 & $20.76 \%$ & $9-13$ & $0-4$ \\
\hline Counter 4 & 8 & 10 & 2 & 0.7657 & 0.1556 & $23.43 \%$ & $8-12$ & $0-4$ \\
\hline Counter 5 & 2 & 8 & 3 & 0.3261 & 0.181 & $67.39 \%$ & $5-11$ & $3-9$ \\
\hline Counter 6 & 3 & 8 & 3 & 0.444 & 0.2047 & $55.60 \%$ & $5-11$ & $2-8$ \\
\hline Counter 7 & 8 & 10 & 2 & 0.7593 & 0.1615 & $24.07 \%$ & $8-12$ & $0-4$ \\
\hline Counter 8 & 3 & 8 & 3 & 0.4362 & 0.2014 & $56.38 \%$ & $5-11$ & $2-8$ \\
\hline Counter 9 & 4 & 8 & 3 & 0.5295 & 0.201 & $47.05 \%$ & $5-11$ & $1-7$ \\
\hline Counter 10 & 3 & 8 & 3 & 0.4364 & 0.1987 & $56.36 \%$ & $5-11$ & $2-8$ \\
\hline Counter 11 & 3 & 8 & 3 & 0.4275 & 0.195 & $57.25 \%$ & $5-11$ & $2-8$ \\
\hline Counter 12 & 1 & 8 & 3 & 0.2336 & 0.1632 & $76.64 \%$ & $5-11$ & $4-10$ \\
\hline Counter 13 & 13 & 14 & 1 & 0.8706 & 0.1068 & $12.94 \%$ & $13-16$ & $0-3$ \\
\hline Counter 14 & 14 & 15 & 1 & 0.883 & 0.0943 & $11.70 \%$ & $14-16$ & $0-2$ \\
\hline Counter 15 & 4 & 8 & 3 & 0.5293 & 0.1995 & $47.07 \%$ & $5-11$ & $1-7$ \\
\hline Counter 16 & 3 & 8 & 3 & 0.4364 & 0.2006 & $56.36 \%$ & $5-11$ & $2-8$ \\
\hline Counter 17 & 4 & 8 & 3 & 0.5256 & 0.203 & $47.44 \%$ & $5-11$ & $1-7$ \\
\hline Counter 18 & 4 & 8 & 3 & 0.5415 & 0.1993 & $45.85 \%$ & $5-11$ & $1-7$ \\
\hline Counter 19 & 2 & 8 & 3 & 0.3401 & 0.1861 & $65.99 \%$ & $5-11$ & $3-9$ \\
\hline Counter 20 & 2 & 8 & 3 & 0.3398 & 0.1875 & $66.02 \%$ & $5-11$ & $3-9$ \\
\hline Counter 21 & 1 & 8 & 3 & 0.2352 & 0.1611 & $76.48 \%$ & $5-11$ & $4-10$ \\
\hline Counter 22 & 5 & 8 & 3 & 0.6071 & 0.1986 & $39.29 \%$ & $6-11$ & $1-6$ \\
\hline Counter 23 & 6 & 9 & 2 & 0.6648 & 0.1851 & $33.52 \%$ & $7-11$ & $1-5$ \\
\hline Counter 24 & 3 & 8 & 3 & 0.4265 & 0.1951 & $57.35 \%$ & $5-11$ & $2-8$ \\
\hline Counter 25 & 8 & 10 & 2 & 0.7521 & 0.1571 & $24.79 \%$ & $8-12$ & $0-4$ \\
\hline Counter 26 & 9 & 11 & 2 & 0.7906 & 0.1432 & $20.94 \%$ & $9-13$ & $0-4$ \\
\hline Counter 27 & 7 & 10 & 2 & 0.717 & 0.175 & $28.30 \%$ & $7-12$ & $0-5$ \\
\hline Counter 28 & 3 & 8 & 3 & 0.4355 & 0.2035 & $56.45 \%$ & $5-11$ & $2-8$ \\
\hline Counter 29 & 6 & 9 & 2 & 0.658 & 0.1905 & $34.20 \%$ & $7-12$ & $1-6$ \\
\hline Counter 30 & 1 & 8 & 3 & 0.2254 & 0.1666 & $77.46 \%$ & $5-11$ & $4-10$ \\
\hline Counter 31 & 3 & 8 & 3 & 0.4478 & 0.2051 & $55.22 \%$ & $5-11$ & $2-8$ \\
\hline Counter 32 & 1 & 8 & 3 & 0.2375 & 0.1699 & $76.25 \%$ & $5-11$ & $4-10$ \\
\hline Counter 33 & 2 & 8 & 3 & 0.3242 & 0.184 & $67.58 \%$ & $5-11$ & $3-9$ \\
\hline Counter 34 & 3 & 8 & 3 & 0.4395 & 0.2014 & $56.05 \%$ & $5-11$ & $2-8$ \\
\hline Counter 35 & 2 & 8 & 3 & 0.3385 & 0.1894 & $66.15 \%$ & $5-11$ & $3-9$ \\
\hline Counter 36 & 4 & 8 & 3 & 0.5297 & 0.2061 & $47.03 \%$ & $5-11$ & $1-7$ \\
\hline Average & 4 & 9 & 3 & 0.5080 & 0.18 & $49.20 \%$ & $6-11$ & $2-7$ \\
\hline
\end{tabular}

From Table 7, we get that the average of the estimators of the actual number of the product sales at a counter with the rate of product sales very low is ranging between 
4-13 pieces with a gap of 1-9 pieces compared to the number of the product sales listed in the report (obtained from the system). The mean percentage of underreporting counts of product sales is $52.88 \%$ or ranged from $32.10 \%$ to $73.66 \%$.

Tabel 7: The Estimators of Model Parameter at Counter with The Very Low Rate of Product Sales

\begin{tabular}{ccccccccc}
\hline Counter & $y_{\boldsymbol{i}}$ & $\widehat{y_{l}^{*}}$ & $\operatorname{std}\left(\widehat{y_{l}}\right)$ & $\widehat{p_{l}^{*}}$ & $\operatorname{std}\left(\widehat{p_{l}^{*}}\right)$ & $\widehat{q_{l}^{*}} x 100 \%$ & $\begin{array}{c}\widehat{y_{l}^{*}} \pm \\
s t d\left(\widehat{y_{l}}\right)\end{array}$ & $\begin{array}{c}{\left[\widehat{y_{l}^{*}} \pm\right.} \\
\left.\operatorname{std}\left(\widehat{y_{l}}\right)\right]-y_{\boldsymbol{i}}\end{array}$ \\
\hline Counter 1 & 1 & 8 & 5 & 0.2634 & 0.2042 & $73.66 \%$ & $3-13$ & $2-12$ \\
Counter 2 & 6 & 9 & 4 & 0.679 & 0.2058 & $32.10 \%$ & $5-13$ & $0-7$ \\
Average & $\mathbf{4}$ & $\mathbf{8}$ & $\mathbf{4}$ & $\mathbf{0 . 4 7 1 2}$ & $\mathbf{0 . 2 0 5}$ & $\mathbf{5 2 . 8 8 \%}$ & $\mathbf{4 - 1 3}$ & $\mathbf{1 - 9}$ \\
\hline
\end{tabular}

\section{Conclusion}

The estimation of the parameters of the regression model for underreported counts performed through by a Bayesian approach and Markov Chain Monte Carlo simulation using Gibbs sampling algorithm, is depend on the determination of burn in period. Determination of burn in period or deleting the first $B$ iterations of the algorithm is done to reduce or avoid the effect of the initial value of the specified parameter. These initial values may affect the posterior summary if they have a huge gap from the highest value of the posterior probability.

Determination of burn in period is done in line with the convergence of algorithm conducted through trace plot, autocorrelation plot and ergodic mean plot. The determination of burn in period is easier using ergodic mean plot than using the trace plot or autocorrelation plot. Ergodic mean plot describes the average simulation values on current iteration. Then, the researcher can more easily determine the first $B$ iterations to be removed. While the trace plot only illustrates the randomness pattern of simulated results and autocorrelation plot only describes the value of autocorrelation between successive iterations. After burn in period is determined, the estimated value of the underreported model parameters is the average of the simulated sample values of each parameter calculated from sample value after burn in period until the last iteration.

The results of estimation clearly showed the percentage of underreporting counts is very high, either on the counter whose sales rate is very high, high, low or very low. it also showed a gap between undamaged product sales and the actual number. The gap is the number of damaged product sales. In order to reduce the risks of the errors on the marketing strategies (see Figure 1), these estimation value can be more useful.

\section{References}

Moreno, E., and Giron, J. (1998). Estimating with incomplete count data A Bayesian approach. Journal of Statistical Planning and Inference, 66(1), 147-159.

Neubauer, G., Djuraš, G., and Friedl, H. (2011). Models for underreporting: A Bernoulli sampling approach for reported counts. Austrian journal of statistics, 4O(1\&2), 8592.

Papadatos, N. (2005). Characterizations of discrete distributions using the Rao-Rubin condition. Journal of statistical planning and inference, 135(1), 222-228. 
Pararai, M., Famoye, F., and Lee, C. (2010). Generalized poisson-poisson mixture model for misreported counts with an application to smoking data. Journal of Data Science, 8(4), 607-617.

Sinaga, U. I. (2013). Faktor-Faktor yang Mempengaruhi Volume Penjualan Sepeda Motor Honda Vario pada PT. Capella Dinamik Nusantara Pekanbaru. Riau University, Pekanbaru.

Winkelmann, R. (1996). Markov chain Monte Carlo analysis of underreported count data with an application to worker absenteeism. Empirical Economics, 21(4), 575587. 